\author{
A. Benaradj, H. Boucherit, O. Hasnaoui \& B. Babali
}

\title{
Phytoécologie de la formation steppique à Drinn (Stipagrostis pungens) dans la région de Naâma (ouest Algérien)
}

\begin{abstract}
Benaradj, A., Boucherit, H., Hasnaoui, O. \& Babali, B.: Phytoécologie de la formation steppique à Drinn (Stipagrostis pungens) dans la région de Naâma (ouest Algérien). — Fl. Medit. 31: 159-171. 2021. — ISSN: 1120-4052 printed, 2240-4538 online.

Phytoecology of the steppic formation at Drinn (Stipagrostis pungens) in the Naâma region (western Algeria). - The steppic plateaus of N-Africa are remarkably affected by silting up and desertification. Sanding favors the installation of psammophytic formations dominated by Stipagrostis pungens, Thymelaea microphylla and Retama raetam on sandy accumulations and wind deposits.

The study focuses on the eco-floristic aspects of the psammophytic grassland steppe of Stipagrostis pengens in the Naâma region (W-Algeria). Its objective is to illustrate the current state and to describe the floristic settlement characteristic of a type of steppe grouping very widespread in the summit Oran plateaus.

The Drinn-dominated grasslands (Stipagrostis pungens) can form a homogeneous steppe very adapted to drought, by physiological reactions, as well as by ecological strategies, facilitating their spreading. The Drinn is loosely distributed in large, dense, branched tufts, more than one meter high on sandy accumulations in an arid climate.

Several parameters are studied to approach the quantitative and qualitative aspects of the floristic settlement. This is followed by a statistical approach, to better interpret the different results obtained. 51 plant species belonging to 14 botanical families and 37 genera of vascular plants have been identified. The results obtained show that the phytoecological inventories are an useful tool to assess and develop natural resources.
\end{abstract}

Key words: steppe, psammophyte, Stipagrostis, ecology, Naâma, Oran.

\section{Introduction}

En Algérie, les écosystèmes steppiques arides sont marqués par une grande diversité paysagère en relation avec une variabilité des facteurs écologiques (Nedjraoui \& Bedrani 2008).

La végétation steppique est caractérisée par un ensemble de communautés qui doivent leurs physionomies, à caractère herbacé et/ou plus ou moins arbustif, à l'abondance soit de graminées cespiteuses à base d'alfa (Stipa tenacissima) ou sparte (Lygeum spartum) ou des chamaephytes tel que l'armoise et le Remth (Hammada scoparia). Ces communautés se développent en touffes espacées dans lesquels la fréquence et le mode de distribution res- 
tent le plus souvent irréguliers. La végétation steppique joue un rôle fondamental dans la structure et le fonctionnement de l'écosystème dont elle constitue une expression du potentiel biologique. Cependant, le couvert végétal naturel est soumis à un double stress édapho-climatique d'une part et au stress anthropozoogène d'autre part (Djebaili 1978; Kaabeche 1990; Moulay \& al. 2011).

La typologie des parcours steppiques peut, valablement, être calquée sur les types physionomiques définis par des végétaux pérennes spontanés dominants à base des graminées (Poacées) qui demeurent des indicateurs écologiques et d'usages (Le Houérou 1995). Les steppes graminéennes à alfa, à sparte et à Drinn jouent à la fois un rôle socio-économique important par la pratique de l'élevage ovin. Ces steppes sont dominées par des graminées pérennes cespiteuses telles que l'alfa (Stipa tenacissima L.), le sparte (Lygeum spartum L.) et certaines autres moins sociables comme le Drinn (Stipagrostis pungens subsp. pungens (Desf.) De Winter.), le zouaï (Stipa lagascae Roem \& Schult., S. barbata subsp. brevipila (Coss. \& Durieu) ou S. parviflora Desf.) et le Chiendent, n'djem (Cynodon dactylon L.) (Le Houérou 1995; Aidoud \& Touffet 1996; Marcenò \& al. 2019). Le Drinn (three-awn grass), Stipagrostis pungens est une Poacee vivace, rhizomenteuse, originaire de l'Afrique du Nord (Quézel \& Santa 1962-1963; Chehma 2006; Dobignard 2008). Les steppes à Drinn constituent des meilleurs pâturages des ovins et des camelins en période disette (Chehma \& al. 2004). La multiplicité des fonctions sociales et le rôle écologique de ces steppes en font un type de paysage important et particulier à comprendre et à gérer (Ghennou 2014).

Le présent travail consiste en une étude phyto-écologique (quantitative et qualitative) du groupement psammophyte à base de Stipagrostis pungens sur les hautes plaines steppiques dans la région sud-oranaise de Naama et cela dans le but de mieux appréhender la dynamique de la végétation et mieux comprendre les facteurs écologiques qui régissent ces formations végétales.

\section{Matériel et méthodes}

Notre choix a porté sur une zone située au sud de la ville de Naâma « appelé communément Souiga ». C'est une zone représentative de la formation steppique à Stipagrostis pungens d'où l'intérêt de ce choix. Elle est située à $17 \mathrm{Km}$ au Sud de la ville de Naâma. Sur le plan géomorphologique la zone présente une pente moyenne de $3 \%$. Elle est localisée entre $33^{\circ} 10^{\prime}$ Nord en latitude et $22^{\circ}$ et $90^{\prime}$ Ouest en longitude et une altitude de $1191 \mathrm{~m}$. Elle constitue un ensemble topographique homogène sans accident topographique majeur. Elle est dominée par la formation psammophytique à base de Drinn (Stipagrostis pungens).

La région d'étude constitue une transition entre le milieu aride des hautes plaines et le domaine aride de l'atlas saharien. Du point de vue climatique, elle appartient à l'étage bioclimatique méditerranéen aride à hiver doux $\left(\mathrm{Q}_{2}=13.38\right.$ et $\left.\mathrm{m}=6.7^{\circ} \mathrm{C}\right)($ Benaradj, 2017). Sur le plan géologique, la région d'étude se localise sur du Pliocène continental, est caractérisée hydrologiquement de faible réseau hydrographique. Sur le plan pédologique, les sols sont de type Sols minéraux brut d'apport. Ce sont des sols squelettiques développés sur les formations superficielles minces. Ils sont représentés par plusieurs types de régosols et lithosols. Les accumulations sableuses sous forme de micro-dunes et de placage sableux, 


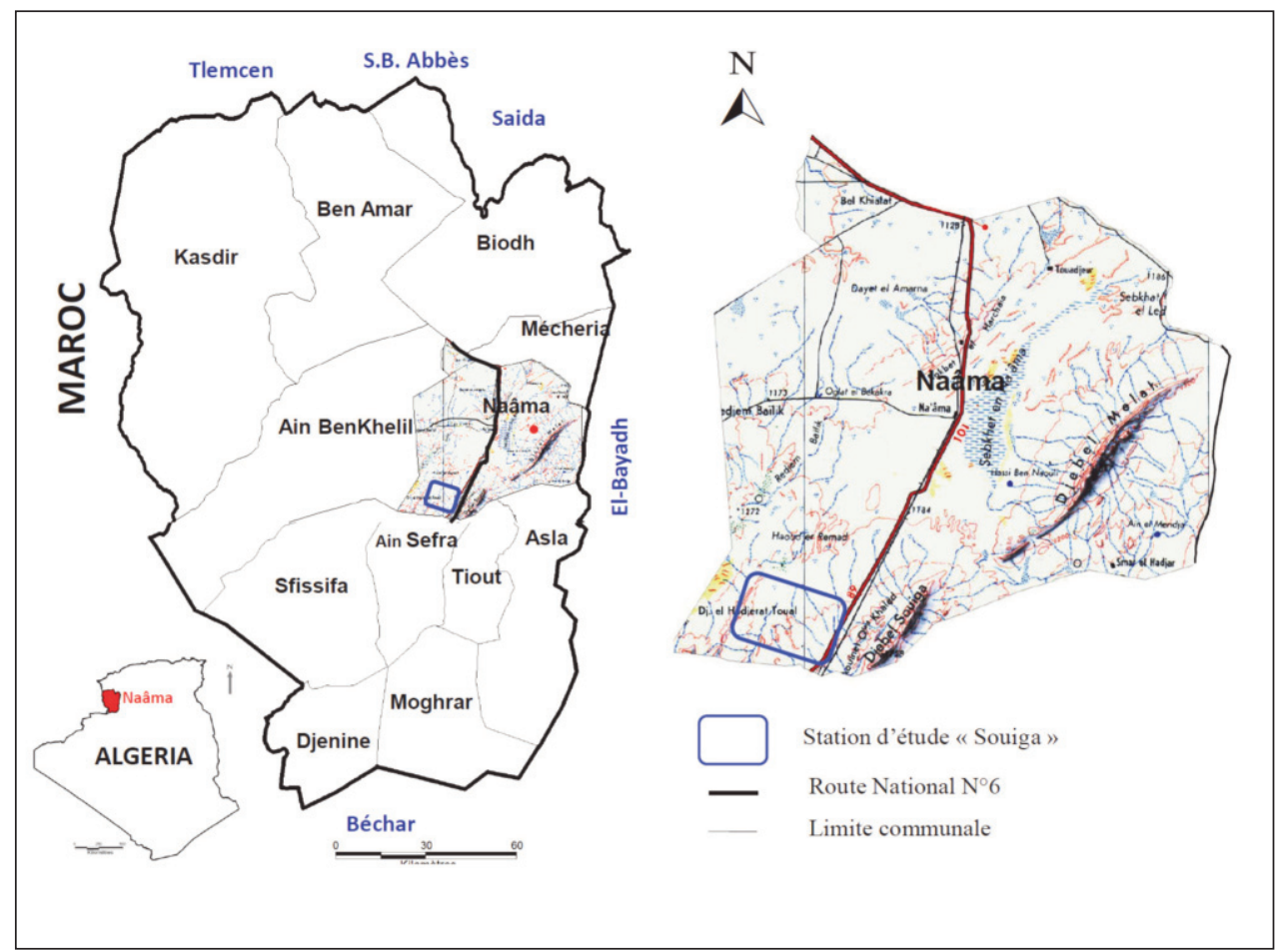

Fig.1. Situation de la zone d'étude (Souiga/Naâma).

correspondent à des sols minéraux bruts, d'apport éolien. Sur le plan phytogéographique, la zone d'étude appartient à la région méditerranéenne qui s'inscrit dans l'empire floral holarctique et au domaine maghrébin-steppique et secteur Hautes Plaines steppiques.

\section{Réalisation des relevées phytoécologique}

Selon Djebaili (1978), l'aire minimale joue un rôle de premier ordre dans la comparaison floristique des relevés. Il est connu que cette aire minimale varie en fonction de chaque groupement végétale. Selon Lemée (1967), l'aire minimale est la plus petite surface nécessaire pour que la plupart des espèces y soient représentaient. C'est un recensement de toutes les espèces rencontrées dans une aire représentative dans le but d'établir une liste floristique des communautés homogènes (Gounot 1969).

Dans cette approche nous nous sommes basés sur les techniques élaborées par Blanquet (1964). Quarante relevés phytoécologiques ont été effectués lors des prospections menées au cours de mois avril et Mai durant le printemps de l'année 2018; saison considérée comme optimale pour les observations du tapis végétal (Electronic Supplementary File 1). L'emplacement de nos relevés floristiques a été choisi d'une façon systématique (au sens de Gounot 1969). Chaque relevé phytoécologique $\left(\right.$ de $\left.100 \mathrm{~m}^{2}\right)$ consiste à faire un inventaire exhaustif de toutes les espèces végétales rencontrées avec leur abondance -dominance et le taux recouvrement estimé (Djebaili 1984; Benaradj 2017). 


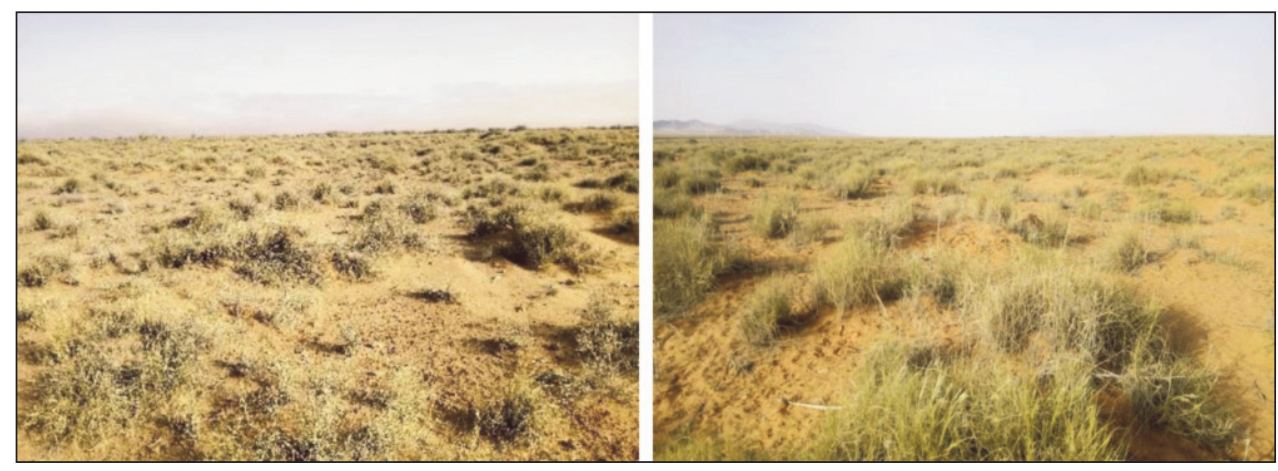

Fig. 2. Payasage steppique à Stipagrostis pungens dans la zone d'étude.

\section{Analyse quantitative de la composition floristique}

Le recouvrement d'une espèce est défini théoriquement comme le pourcentage de la surface du sol qui serait recouverte, si on projetait verticalement sur le sol les organes aériens des individus de l'espèce (Gounot 1969).

La richesse floristique d'un territoire est le nombre total d'espèces qu'il renferme, cette richesse floristique est en générale d'autant plus élevée que la surface du territoire est plus grande, mais croit naturellement moins vite que la superficie considérée. Dans notre cas nous avons utilisé l'échelle de Daget et Poissonet (1991) et qui comme suit:
Raréfiée: < de 5 espèces
Très pauvre: de 6 à 10 espèces
Pauvre de 11 à 20 espèces
Moyenne: de 21 à 30 espèces
Assez riche de 31 à 40 espèces
Riche: de 41 à 60 espèces
Très riche: de 61 à 75 espèces

\section{Analyse qualitative (recouvrement et richesse floristique)}

Plusieurs paramètres ont été pris en considération pour cerner l'aspect quantitatif de la végétation. Ces paramètres nous ont permis de recenser les espèces végétales et de dégager la structure de la végétation sur plusieurs plans:

- Plan biologique selon Raunkiaer (Raunkiaer 1934) et Daget (1980),

- Plan systématique selon les travaux de Quézel \& Santa (1962-1963); Ozenda (1977), Fennane \& Ibn Tattou (1999); Fennane \& al. (2007) et l'APG III (Angiosperm Phylogeny Group 2009).

- Plan biogéographique selon Quéze 1\& Santa et Le Houérou (1995).

\section{Traitement des données floristiques}

Les explications sont étayées par une Analyse Factorielle des Correspondances (A.F.C) combiné à la Classification Hiérarchique Ascendante (C.A.H) afin de mieux cerner les facteurs écologiques prépondérants. Cette analyse statistique fine, nous a permis de connaitre 
les divers facteurs qui interviennent dans la distribution et la répartition de la formation steppique psmmophytique à Drinn (Stipagrostis pungens) dans la région d'étude.

\section{Résultats et discussion}

\section{Aspect qualitatif}

Le recouvrement moyen de la végétation dans la station d'étude est très ouvert. Il comprise entre 5 à $25 \%$, cela peut être expliqué par le type de formation steppique se développent sur un milieu dunaire (sableux). Le milieu dunaire de cette formation à Stipagrostis pungens se caractérise par un faible recouvrement. D'après Daget \& Godron (1995), le taux de recouvrement en tant qu'indicateur de dégradation est indiscutable du moins sur le plan environnemental dans des régions similaires menacées par la désertification.

De point de vue richesse floristique, on a enregistré plus de 51 espèces dans les dans la station de Souiga. D'après Daget \& Poissonet (1991), la station est riche en espèces, parce que le nombre d'espèces inventorié durant notre investigation est supérieur à 40 espèces. D'après Aidoud (1989), la richesse floristique en zone aride dépend essentiellement des espèces annuelles, des conditions du milieu et de la corrélation de l'ensemble des caractères (climat, édaphisme et exploitation). Lemée (1953), lie la richesse floristique des biotopes aux déterminismes édaphiques (texture et nature chimique du sol) et anthropozoïque qui provoque l'enrichissement des sols en matières azotées à travers le surpâturage.

Selon Kerroum (2014), la variation de la composition floristique est sous l'effet de l'action synergique de l'aridité et de l'anthropique. En effet, l'aridité du climat, la sécheresse, l'ensablement et la désertification de la zone d'étude ont un impact négatif sur la richesse floristique (Boucherit 2018).

\section{Aspect qualitatif de la composition floristique}

L'inventaire floristique permet de recenser 51 taxons, répartis en 15 familles et 44 genres. Donc, le cortège floristique cache une phytodiversité hétérogène sur plan quantitatif et qualitatif; celle-ci est liée à la position géographique, nature édaphique, climatique et l'action anthropique.

Sur le plan systématique, les espèces représentées sont variables, la répartition des familles est hétérogène. D'après la figure 2.b, on note une nette dominance de la famille des Poaceae (avec 8 genres et 10 espèces), suivis par les Astéraceae (6 genres et 7 espèces), des Fabaceae (avec 5 genres et 6 espèces), des Lamiaceae ( 3 genres et 3 espèces) puis respectivement suivis par les familles bigénériques (Amaranthaceae, Brassicaceae et Borraginaceae, Caryophyllaceae (2 genres et 2 espèces). Les familles restantes sont monospécifiques: Cleomaceae, Geraniaceae, Plantaginaceae, Ranunculaceae, Tamaricaceae, Thymeliaceae.

Sur le plan biologique la liste floristique établie, montre la prédominance des thérophytes sur les autres formes avec 19 espèces soit 45\%. Cette prédominance est caractéristique de la végétation des régions arides qui s'adaptent qu'offre les milieux steppiques et sahariens.

Sur le plan biogéographique, il ressort la prédominance de l'élément méditerranéen (25\%); ce qui confirme l'affinité méditerranéenne de la flore de la région qui s'agence entre les deux ensembles les hautes plaines steppique et l'atlas saharien au Sud. Pour les 

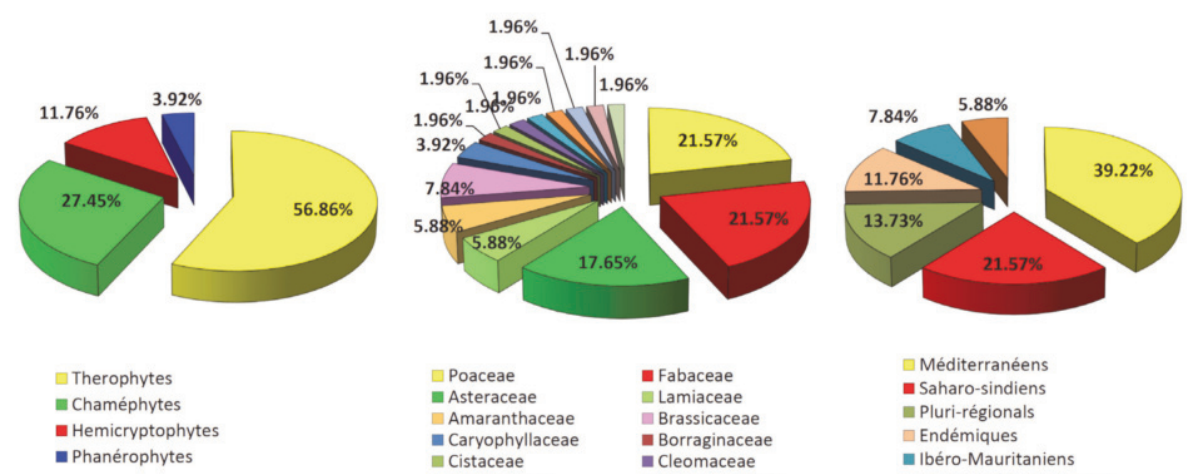

a- Biologique

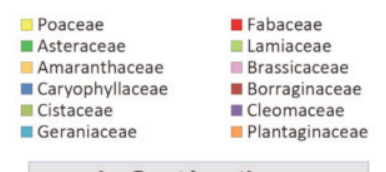

b- Systématique

Méditerranéens

a Saharo-sindiens

- Pluri-régionals

Endémiques

- Ibéro-Mauritaniens

- Macar-Méd.-Irano-Tour

c- Biogéographique

Fig.3. Caractérisation quantitative (a- biologique, b- systématique, c- biogéographique).

autres éléments biogéographique on note la forte présence de l'élément Saharo-sindien (15\%), suivi par l'endémique (12.5\%), le Saharien (10\%), Pluri régionale avec $(12.5 \%)$. Les autres éléments ont une présence réduite c'est le cas de: Ibéro Mauritanien $(5 \%)$, Macar-Méd.-Irano-Tour (7.5\%).

Le groupement graminéen à Stipagrostis pungens présente une importante diversité floristique sur le plan biologique, systématique et biogéographique. Les types biologiques et phytogéographiques permettent d'apprécier les différentes stratégies d'adaptation de vie et une large répartition géographique.

L'inventaire floristique permet de recenser 51 taxons, répartis en 15 familles et 44 genres. Donc, le cortège floristique cache une phytodiversité hétérogène sur plan quantitatif et qualitatif; celle-ci est liée à la position géographique, nature édaphique, climatique et l'action anthropique.

Sur le plan systématique, la forte proportion des Poaceae dans la zone d'étude peut s'expliquer par le fait que ces taxons possèdent une très grande possibilité de tallage et une plus grande vitesse de repousse après le passage des troupeaux. Les Poaceae et les Fabaceae offrent un potentiel fourrager très important; ce qui favoriserait l'exploitation des parcours à des fins pastorales (Yoka \& al. 2013). Les familles des Asteraceae, Poaceae présentent un plus grand pouvoir d'adaptation aux changements des conditions écologiques et aux pressions anthropiques (surpâturage) dans ces milieux.

Sur le plan biologique, la prédominance des thérophytes est caractéristique de la végétation des régions arides qui s'adaptent qu'offre les milieux steppiques et sahariens. Plusieurs auteurs soulignent que les thérophytes dépendent directement des précipitations (Quézel 1965; Barkoudah \& Van Der Sar 1982; Carriere 1989; Grouzis 1992; Monod 1992). D'autres lient leur présence à l'état de la surface du sol (Negre 1966; Kadi-Hanifi 2003). Daget (1980) et Barbero \& al. (1990) soulignent que la thérophytie est une stratégie d'adaptation à la sécheresse. Grime (1977), lie la présence des thérophytes aux perturbations des milieux. Guinochet \& Quézel (1954) indiquent que la présence de sable même en 
couche réduite dans les habitats entraine le développement des psammophytes, surtout annuelles. D'un autre coté la chamephytisation semble très lie à la dégradation d'origine anthropique du milieu avec la prolifération des espèces non palatables (Thymelaea microphylla Coss., Atractylis serratuloides Sieber ex Cass., Echinops spinosus L., etc.). Sur le plan biogéographique, d'après ces différents éléments, il ressort que le milieu d'étude est diversifié sur le plan phytogéographique. Cette diversité est liée d'après Quézel (1995) à deux séries majeures de causes: les changements climatiques qui provoquent la migration des flores et le transport à longues distances par les vents et les oiseaux.

\section{Traitement des données}

L'A.F.C. réalisée sur une matrice de 40 relevés floristiques et 51 espèces, nous a permis de dégager les divers facteurs qui influent sur le développement des espèces accompagnatrices de la steppe à Stipagrostis pungens.

Tableau 1. Valeur propre et taux d'inertie (\%) de l'A.F.C.

\begin{tabular}{|l|c|c|c|c|}
\hline Plan factoriel & Axe 1 & Axe 2 & Axe 3 & Total \\
\hline Valeurs propres & 0,526 & 0,094 & 0,087 & 0,708 \\
\hline Taux d'inertie (\%) & 14,728 & 2,645 & 2,446 & 19,820 \\
\hline Cumul (\%) & 14,728 & 17,373 & 19,819 & \\
\hline
\end{tabular}

Dans cette analyse, seul le plan factoriel (1/2) semble être le plus intéressant, puisqu'il absorbe le maximum d'informations et explique $17.373 \%$ de la variation totale (Tab. 1). Les valeurs propres de deux axes ( 1 et 2 ) sont respectivement de 0,526 et 0,094. Elles témoignent d'une structuration hétérogène du nuage. Les espèces sont distribuées selon un nuage centré sur l'origine des axes dans le plan $1 / 2$, qui se traduit par une assez nette séparation spatiale des relevés, ceci a permis d'individualiser 3 groupes de relevés (Fig.4 et Fig.5).

Le CAH des relevés floristiques a permis de distinguer les différents groupes d'espèces les plus caractéristiques du groupement à Stipagrostis pungens. Les résultats sont présentés sous forme de dendrogramme regroupant les relevés selon le degré de similitude. Celle-ci est estimée par un critère de proximité ou de distance choisi à priori (Kaabèche 1990).

Dans notre cas (Fig.4), les 29 espèces les plus représentatives ont été prises en considération, car elles sont largement répandues et donc les plus dominantes et les plus abondantes dans les relevés floristique réalisés au niveau du groupement à Stipagrostis pungens. Pour les autres espèces qui ne figurent pas dans la dendrogramme (Fig.4), ces espèces ont une fréquence très réduite (à faible similarité et/ou faible présence). Elles sont non représentatives numériquement et écologiquement. Elles peuvent être considérées soit comme des espèces accidentelles ou rares, soit sont des espèces notées hors relevés effectués.

L'information donnée par les deux axes de l'analyse factorielle des correspondances (AFC) (plan F1/F2=18\%), suivie de l'analyse hiérarchique des correspondances (C.A.H., Fig. 4), nous a permis d'identifier la présence de trois groupements de végétaux à savoir: 


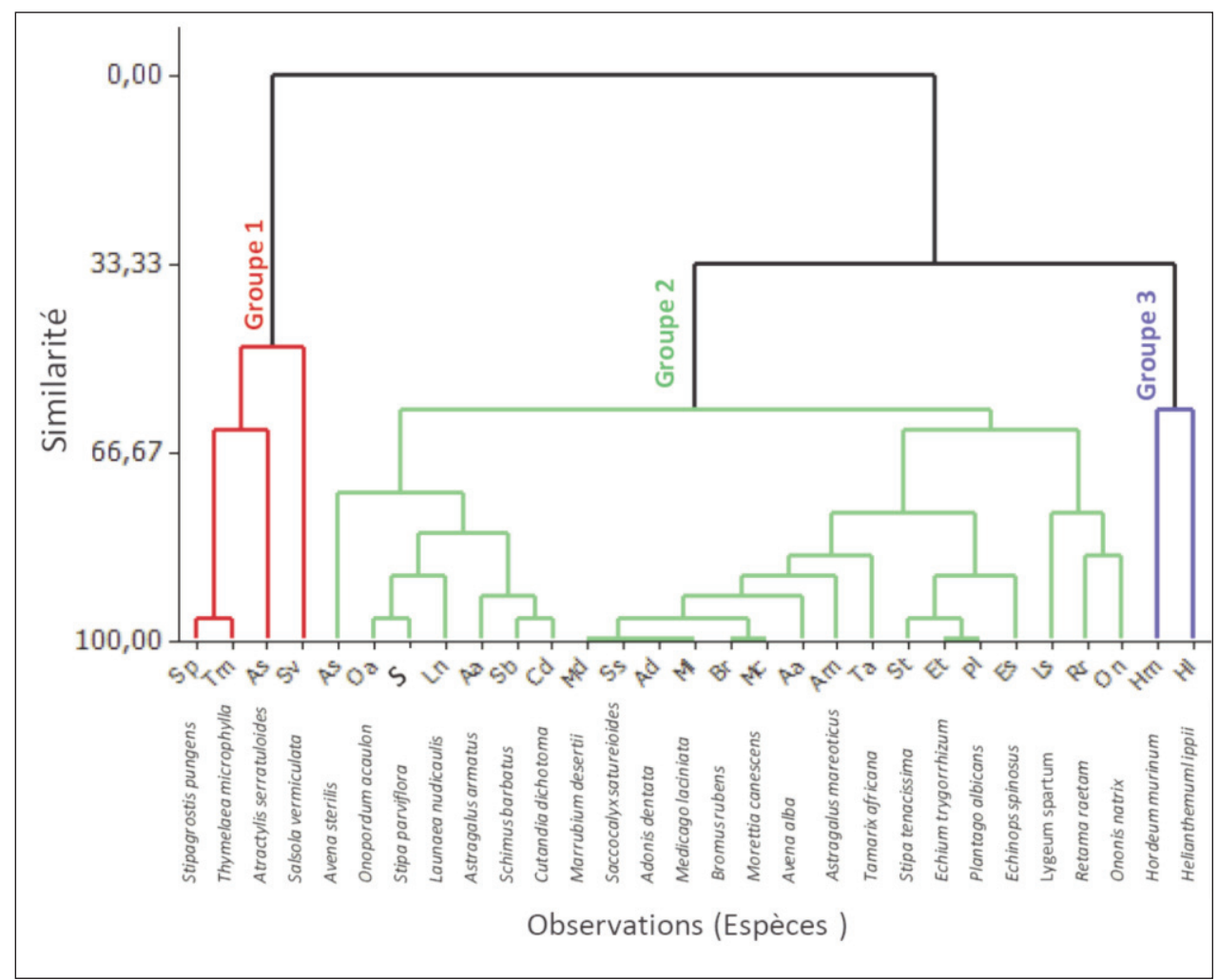

Fig. 4. Dendrogramme de la C.A.H de la matrice totale des espèces.

-Groupe 1: à base de steppe graminéennes à Stipagrostis pungensen association avec les espèces de Thymelaea microphylla Coss., Atractylis serratuloides Sieber ex Cass., Salsola vermiculata L., où s'individualise nettement et regroupe plus $90 \%$ des relevés floristique.

- Groupe 2: ce groupe est constitué par le cortège floristique suivant: Retama raetam (Forssk.) Webb, Atractylis serratuloides Cass., Lygeum spartum L., Stipa tenacissima L., Echium trygorrhizum Pomel, Plantago albicans L., Avena alba Vahl, Stipa parviflora Desf., Schimus barbatus L., Cutandia dichotoma (Forssk.) Trab., Bromus rubens L., Morettia canescens Boiss., Ononis natrix L., Astragalus mareoticus Delile., Launaea nudicaulis Hook.f., Astragalus armatus subsp. numidicus (Murb.) Emb. \& Maire, Onopordum acaulon L., Saccocalyx satureioides Coss. \& Durieu., Marrubium desertii Noé ex Coss., Echinops spinosus L., Adonis dentata Delile., Medicago laciniata L., etc.

- Groupe 3 « Hm et Hl»: est dominé de l'espèce Hordeum murinum L., leur présence presque dans tous les relevés floristiques, ainsi la présence de l'espèce ligneuse Helianthemum lippii L.

L'information donnée par les deux axes de l'analyse factorielle des correspondances (AFC) (plan F1/F2=18\%), suivie de l'analyse hiérarchique des correspondances (C.A.H., Fig. 4), nous a permis d'identifier la présence de trois groupements de végétaux à savoir: 


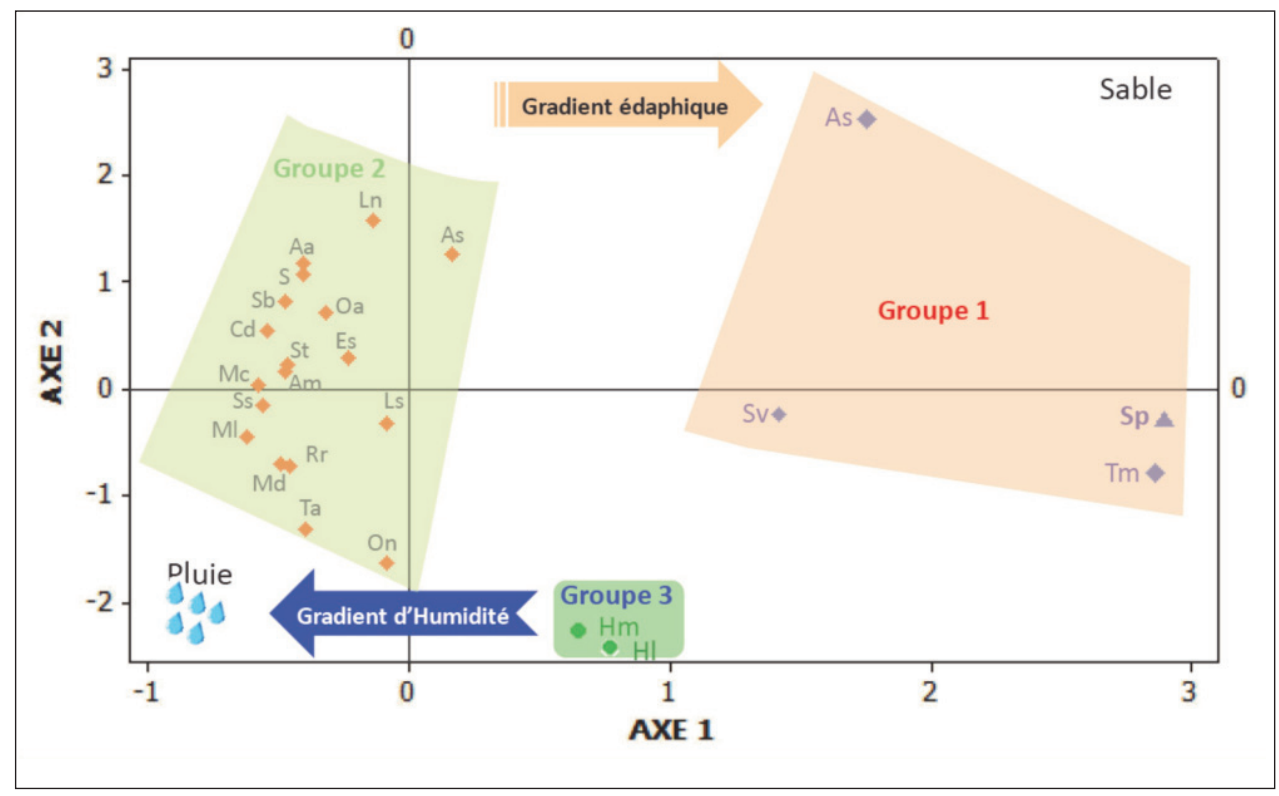

Fig 5. Plan Factoriel du premier/deuxième axe des espèces accompagnatrices de la steppe à Stipagrostis pungens.

-Groupe 1: Cet ordre à Stipagrostis pungens se rattache à l'ordre des Aristidetalia pungentis (Guinochet, 1952) et de l'alliance Aristidion pungentis au sens de Géhu, Kaabèche \& Gharzouli (1993). En effet, Gauthier Pilters (1972); Chehma \& longo (2004); rapportent que les peuplements de Drinn (Stipagrostis pungens) constituent d'immenses peuplements homogènes dans les massifs dunaires. Dajoz (1970) et Djebaili (1978) rapportent que les sols sableux sont essentiellement caractérisés par la dominance de Stipagrostis pungens. Cette steppe graminéenne s'installe également sur les glacis dès que l'accumulation sableuse devient supérieure à $50 \mathrm{~cm}$ mais n'atteint cependant pas une forme aussi sociale que le sparte ou l'alfa. L'installation du Drinn a été observée suite à la destruction de l'alfa et à de fortes accumulations de sable dans la station d'étude.

- Groupe 2: Ces espèces sont généralement liées à l'humidité des formations pré-steppiques. Ces dernières sont toutes représentatives de groupements à caractère psammophile liés aux dunes vives relevant des Aristidetalia (Guinochet 1952). Elles sont concentrées au centre des axes. Cette concentration est expliquée par le fait que l'ensemble des espèces citées, trouvent l'humidité optimale pour leurs développements.

- Groupe 3: il est dominé par les 2 espèces, espèce ligneuse (Helianthemum lippii L.) et l'espèce herbacée (Hordeum murinum L.) est très fréquente partout dans les zones arides méditerranéennes. Cela indique que l'Hordeum murinum à stratégie « $\mathrm{r}$ », caractéristique d'espèces qui ont une grande vitesse de multiplication, ce qui leur permet, sous certaines conditions favorables, d'occuper l'espace. 
Cet axe du pôle négatif au pôle positif oppose les espèces xérophytes à celles qui poussent sur des biotopes humides. La matrice de corrélation montre que l'axe 1 est très corrélé au sable du côté négatif (Thymelaea microphylla Coss., Atractylis serratuloides Coss. \& Durieu., Schismus barbatus L., Salsola vermiculata L., Malcolmia aegyptiaca Spreng., Astragalus armatus Willd., Euphorbia guyoniana Boiss. \& Reut.), alors que l'axe 2 est fortement corrélé du côté positif à l'humidité.

Ozenda (1954) indique que le Faciès à Drinn (Stipagrostis pungens) est physionomiquement dominant où pousse au niveau des grandes accumulations sableuses dont le sable est faiblement fixé, cette espèce est caractéristique des milieux à accumulations sableuses.

Selon Kaabèche (1990), le Faciès à Drinn présente un déterminisme édaphique lié à un gradient d'ordre textural. Il est plus fréquent en zones arides steppiques et présahariennes sur les accumulations sableuses. Il est dominé par les espèces psmmophytes telles que Thymelaea microphylla Coss., Salsola vermiculata L. et Avena sterilis L.. Ces espèces sont distribuées largement dans les dunes et s'adaptent à leur environnement par des réactions physiologiques, ainsi que par des stratégies écologiques, permettant de faire face aux pénuries d'eau en évitant ou en tolérant le stress (Djebaili 1984; Gamoun \& al. 2018). Cela s'explique par le caractère psammophile de ces espèces qui se développent aisément sur substrat sableux et s'adaptent bien à la sécheresse et résistent à la rigueur des conditions climatiques (Le Houérou 1995; Hellal \& al. 2004). De même Le Houérou (1995) et Ozenda (2004) rapportent que les sols sableux dans les steppes nord africaines sont généralement dominés par les Poaceae. Cette famille assure un grand rôle dans la fixation des dunes.

Les résultats font ressortir une dynamique régressive de la végétation qui se traduit par un changement de composition floristique et édaphique (accumulations sableuses), accompagné par le remplacement de l'alfa et de sparte par le Stipagrostis pungens, Thymelaea microphylla Coss. et Retama raetam (Forssk.) Webb, toutes ces espèces étant favorisées par l'ensablement de surface.

\section{Conclusion}

La région de Nâama est dominée par une steppe graminéenne à base de Stipagrostis pungens. C'est une formation basse, très ouverte, xérophyte, physionomiquement sous forme des touffes dominantes sur des accumulations sableuses d'origine éolienne. Elle apparaît après une dégradation des formations à base d'alfa (Stipa tenacissima) et/ou de sparte (Lygeum spartum). Le faciès à Stipagrostis pungens est très adapté aux conditions arides et possède une puissance de prolifération importante puisqu'il peut former à lui seul des vastes steppes homogènes.

Devant les conditions actuelles des changements globaux (synergie climato-anthropique) ces formations jouent un rôle non négligeable dans la limitation de l'avancé du désert, chose qui préoccupe les gestionnaires et les scientifiques. Ces formations végétales sont aussi des sources d'alimentation pour le cheptel camelin (Chehma \& al. 2004).

A travers ses différentes caractéristiques éco-physiologiques, Stipagrostis pungens offre de multiples possibilités de valorisation sur le plan écologique, agropastoral, artisanal, ethnobotanique, alimentaire et technologique (Ferhi \& al. 2014). 


\section{Références}

Aïdoud, A. 1989: Contribution à l'étude des écosystèmes steppiques pâturés des hautes plaines Algéro-oranaises (Algérie). - Thèse Doct. U.ST.H.B. Alger.

— \& Touffet, J. 1996: La régression d'alfa (Stipa tenacissima L.), graminées pérennes, un indicateur de désertification des steppes algériennes. - Cahier «Sécheresse» 7(3): 187-193.

APG III., 2009. An update of the Angiosperm Phylogeny Group Classification for the orders and families flowering plants: APG III. - Bot. J. Linn. Soc. 161: 105-121. https://doi.org/10.1111/j.1095-8339.2009.00996.x

Barkoudah, Y. \& Van Der Sar, D. 1982: L'Acacia raddiana dans la région de Béni-Abbés (Algérie). - Bull. Soc. Hist. Nat. Afr. Nord 70(1-4): 79-121.

Barbero, M., Bonin, G., Loisel, R. \& Quézel, P.1990: Changes and disturbances of forest ecosystems caused by human activities in the Western part of Mediterranean Basin. - Vegetatio 87: 151-173.

Benaradj, A. 2017: Étude phyto-écologique des groupements à Pistacia atlantica Desf. dans le sud Oranais (Sud-Ouest algérien). - Thèse Doctorat en Foresterie. Département des Ressources Forestières. Faculté des Sciences de la nature de la vie et sciences de la terre et de l'univers. Université Abou Bakr Belkaid Tlemcen.

Braun-Blanquet, J. 1964: Pflanzensoziologie, Grundzüge der Vegetationskunde, $3^{\text {rd }}$ Ed. - Berlin. http://dx.doi.org/10.1007/978-3-7091-8110-2

Boucherit, H. 2018: Etude ethnobotanique et floristique de la steppe à Hammada scoparia (Pomel) dans la région de Naâma (Algérie). - Thèse Doctorat en Agronomie. Département des Sciences agronomiques. Faculté des Sciences de la nature de la vie et sciences de la terre et de l'univers. Université Abou Bakr Belkaid Tlemcen.

Carriere, M. 1989: Les communautés végétales sahéliennes en Mauritanie (région de Kaédi); analyse de la reconstitution annuelle du couvert herbacé. - Thèse Doct. Sc., Univ. Paris Sud (Orsay).

Chehma, A. 2006: Catalogue des plantes spontanées du Sahara septentrional algérien. - Montpellier.

— \& Longo, H. F. 2004: Nitrogenous record and weight gains in dromedary and sheep fed with date palm, barley straw, and drinn "Aristida pungens" by-products. - Cah. Agric. 13(2): 221-226.

—, Gaouar, A., Semadi, A. \& Faye, B. 2004: Productivité fourragère des parcours camelins en Algérie: cas des pâturages a base de «Drinn» Stipagrostis pungens (Desf.). - Sci. Technol. 21: 45-52. http://revue.umc.edu.dz/index.php/c/article/view/1328

Djebaili, S. 1984: Steppe algérienne, phytosociologie et écologie. - Alger.

Daget, P. 1980: Sur les types biologiques en tant que stratégie adaptative. (Cas des thérophytes). Pp. 89-114 in: Recherches d'écologie théorique, les stratégies adaptatives. - Paris.

— \& Poissonet, J. 1991: Prairies permanentes et pâturages. Méthodes d'étude. Montpellier.

— and Godron, M. 1995: Pastoralisme: Troupeaux, espaces et sociétés. - Hatier, Aupelf, Uref.

Dajoz, R. 1970: Précis d'écologie. - Paris.

Djebaili, S. 1978: Recherches phytosociologiques et phytoécologiques sur la végétation des Hautes plaines steppiques et de l'Atlas saharien ». - Thèse Doct. Univ. Montpellier.

Dobignard, A. 2008: Index synonymique et bibliographique de la flore d'Afrique du Nord. Base de données des plantes à fleurs d'Afrique. - Genève.

Fennane, M. \& Ibn Tattou, M. 1999: Observations sur la flore vasculaire endémique, rare ou menacée du Maroc. - Fl. Medit. 9: 13-124.

Ferhi, F., Das, S., Moussaoui, Y., Elaloui, E. \& Garrido Yanez, J. 2014: Paper from Stipagrostis pungens. - Industr. Crops Prod. 59: 109-114. https://doi.org/10.1016/j.indcrop.2014.05.015

—, Ibn Tattou, M., Ouyahya, A. \& El Oualidi, J. 2007: Flore pratique du Maroc, 2. - Rabat.

Gauthier-Pilters, H. 1972: Observation sur la consommation d'eau du dromadaire en été dans la région de Beni Abbés (Sahara Nord Occidental). - Bull. I. F. A. N. T., sér. A, 34(1): 219-259. 
Gehu, J-M., Kaabeche, M. \& Gharzouli, R. 1993: Une remarquable toposéquence phytocoenotique en bordure du Chott El Hodna (Algérie). - Fragm. Flor. Geobot. Suppl. 2(2): 513-520.

Ghennou, S. 2014: Contribution à une étude dynamique de Stipa tenacissima L dans le Sud-ouest de la région de Tlemcen. - Mémoire Magister en Ecologie, Département d'Ecologie et Environnement. Faculté des Sciences de la nature de la vie et sciences de la terre et de l'univers. Université Abou Bakr Belkaid Tlemcen.

Gamoun, M., Ouled Belgacem, A. \& Louhaichi, M. 2018. Diversity of desert rangelands of Tunisia. - P1. Diversity 40(1): 1-9. https://doi.org/10.1016/j.pld.2018.06.004

Gounot, M. 1969: Méthodes d'étude quantitatives de la végétation. - Paris.

Guinochet, M. 1952: Contribution à l'étude phytosociologique du sud tunisien. - Bull. Soc. Hist. Nat. Afr. Nord. 42: 131-153.

— \& Quézel, P. 1954: Reconnaissance phytosociologique autour du Grand Erg occidental. - Trav. Inst. Rech. Saharienne 12: 11-27.

Grime, J. P. 1977: Evidence for the existence of three primary strategies in plants and its relevance to ecological and evolutionary theory. - Amer. Naturalist 111: 1169-1194.

Grouzis, M. 1992: Germination et établissement des plantes annuelles sahéliennes. - Pp. 245-263 in Le Floch, E., Grouzis, M., Cornet, A., Bille, J.-C. (eds), L'Aridité une Contrainte au Développement. - Montpellier.

Hellal, B., Benseddik, B., Ayad, N. \& Benhassaini, H. 2004: La régénération dans la steppe du sud oranais en Algérie occidentale. - Sécheresse 15(1): 1-7.

Nedjraoui, D. \& Bedrani, S. 2008: La désertification dans les steppes algériennes: causes, impacts et actions de lutte. Revue électronique en sciences de l'environnement. - VertigO 8: 7-15.

Kaabeche, M. 1990: Les Groupements Végétaux de la Région de Bou-Saada. Contribution à la Synsystématique des Groupements steppiques du Maghreb. - Thèse de Doctorat d'Université. 2 Vol., Université de Paris-Sud, Centre d'Orsay, France.

Kadi-Hanifi, H. 2003: Diversité biologique et phytogéographique des formations à Stipa tenacissima L. de l'Algérie. - Sécheresse 14(3): 169-179.

Kerroum, Z. 2014: Contribution à l'Etude phytoécologique des groupements à matorrals de Bouriche (Daïra de Youb Wilaya de Saida). - Mémoire de Master en Master en Ecologie et Environnement. Département de Biologie. Faculté des Sciences. Université Dr. Tahar Moulay, Saïda.

Le Houérou, H. N. (1995). Considérations biogéographiques sur les steppes arides du Nord de l'Afrique (A). - Sécheresse 6(2): 167-82.

Lemée G., 1953: Sur la végétation postglaciaire du Lévezou d'après l'analyse pollinique. - Bull. Soc. Bot. Fr. 100: 26-29.

- 1967: Précis de biogéographie. - Paris.

Marcenò, C., Guarino, R., Mucina, L., Biurrun, I., Deil, U., Shaltout, K., Finckh, M., Font, X. \& Loidi, J. 2019: A formal classification of the Lygeum spartum vegetation of the Mediterranean Region. - Appl. Veg. Sci. 22: 593-608. https://doi.org/10.1111/avsc.12456

Monod, T. 1992. Du désert. - Sécheresse 3(1): 7-24.

Moulay, A., Benabdeli, K. \& Morsli, A. 2011: Contribution à l'identification des principaux facteurs de dégradation des steppes a Stipa tenacissima du sud-ouest Algérien. - Mediterranea, Ser. Estud. Biol., 22: 149-188. http://dx.doi.org/10.14198/MDTRRA2011.22.06

Negre, R. 1966: Les thérophytes. - Mém. Soc. Bot. Fr. 113: 92-108.

Ozenda, P. 1954: Observation sur la végétation d'une région semi- aride: les hauts plateaux du sud Algérien. - Bull. Soc. Hist. Nat. D'Afr. du Nord, Alger, 45(3-4): 189-223.

- 1977: Flore du Sahara, $2^{\text {ème }}$ Ed. - Paris.

— 2004: Flore et végétation du Sahara. - Ecol. Medit. 30(2): 1-247.

Quézel, P. 1965: La végétation du Sahara, du Tchad à la Mauritanie. - Paris. 
- 1995: La flore du bassin méditerranéen: origine, mise en piace, endémisme. - Ecol. Medit. 20(1/2): 19-39.

— \& Santa, S. 1962-1963: Nouvelle flore de l'Algérie et des régions désertiques méridionales, 1-2. - Paris.

Raunkiaer, C. 1934: The Life Forms of Plants and Statistical Plant Geography. - London.

Yoka, J., Loumeto, J. L, Djego, J., Vouidibio, J. \& Epron, D. 2013: Évaluation de la diversité floristique en herbacées des savanes de la cuvette congolaise (République du Congo). - Afrique Sci. 9(2): 110-123.

Adresses des auteurs:

Abdelkrim Benaradj ${ }^{1}$, Hafidha Boucherit ${ }^{2}$, Okkacha Hasnaoui ${ }^{3} \&$ Brahim Babali $^{4}$, ${ }^{1}$ Laboratoire Gestion durable des ressources naturelles des zones arides et semi-arides, Centre Universitaire Salhi Ahmed de Naâma (Algérie). Email: benaradj@cunivnaama.dz

${ }^{2}$ Laboratoire Gestion durable des ressources naturelles des zones arides et semi-arides, Centre Universitaire Salhi Ahmed de Naâma (Algérie). Email: boucherit@cuniv-naama.dz

${ }^{3}$ Laboratoire d'Ecologie Végétale et Gestion des Ecosystèmes Naturels (U Tlemcen). Université Dr Tahar Moulay Saida - Algérie (Algérie), Email: okhasnaoui2001@yahoo.fr

${ }^{4}$ Laboratoire d'Ecologie Végétale et Gestion des Ecosystèmes Naturels, Université Abou Bakr Belkaid de Tlemcen (Algérie).Email: miharb_babali@hotmail.fr 
